\title{
The Effects of Relative Muscle Strength and Flexibility on Injury in Running
}

\author{
James Hou ${ }^{1}$ and Alfred Renaud" \\ ${ }^{1}$ St. George's School, Vancouver, BC, Canada \\ \#Advisor
}

\section{ABSTRACT}

When exercising, physical injury is almost inevitable. Although there are a multitude of practices to avoid injury, a large portion of luck is required to minimize injury proneness. Thus, in this paper, with the aid of a public dataset gait kinetics and kinematics, flexibility and strength are tested against the boolean value of injury to conduct a linear binary regression model.

\section{Introduction}

In all physical activity, it is imperative that injury be avoided to maintain a plethora of routines from training intensity and volume to sleeping and eating habits; however, injury is almost inevitable in all athletes. This inevitability is caused due to the almost infinite number of variables that must be accounted for. In this paper, relative muscle strength and flexibility (both being double values) will be observed against past injury (a boolean value) and future injury proneness. Furthermore, the outcome of the tests conducted will further depict the variability of injury that is not correlated with muscle strength and flexibility. The smaller the angle of flexibility or lower the relative muscle strength, the more prone one is to injury and the higher chance of a previous injury due to certain muscle groups only managing to take a certain varying amount of stress before they are damaged to the point of injury.

\section{Methods and Procedure}

This paper's raw data is based off a public dataset of running biomechanics and the effects of running speed on lower extremity kinematics and kinetics (Fukuchi et al,. 2017). The study was conducted at the Laboratory of Biomechanics and Motor Control at the Federal University of ABC (UFABC) The data collection was performed by experienced physiotherapist researchers.

The study analyzed a convenience sample of 28 subjects who were recruited through posted flyers, advertisement on the BMClab Internet homepage, and social media. The inclusion criteria included being a regular runner with a weekly mileage greater than $20 \mathrm{~km}$, a minimum average running pace of $1 \mathrm{~km}$ in $5 \mathrm{~km}$ in 5 minutes during $10 \mathrm{~km}$ races, and familiarity and comfort with running on a treadmill. 


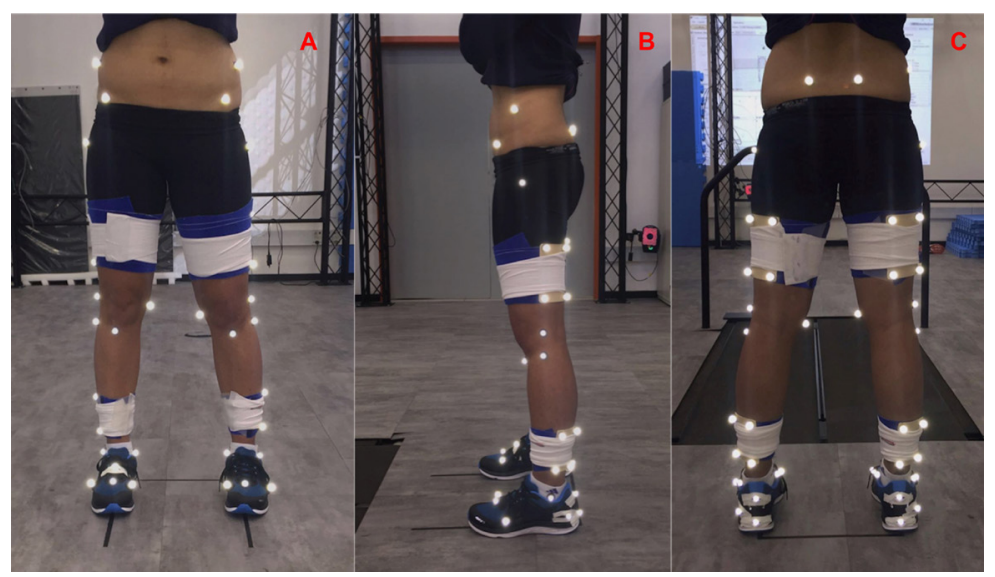

Figure 1. The technical and anatomical marker set protocol during an anatomical calibration trial in the anterior (A), lateral (B) and posterior (C) views (Fukuchi et al,. 2017).

The lower extremity kinematics and kinetics of 28 male regular runners (ranging from short to long distances) were observed and collected using a three-dimensional real time motion-capture system and a standard instrumented treadmill. Subjects were asked to run at $2.5 \mathrm{~m} / \mathrm{s}, 3.5 \mathrm{~m} / \mathrm{s} 4.5 \mathrm{~m} / \mathrm{s}$ while wearing neutral shoes.

It is important to note that the paper that provided the raw data was a public dataset with no research question asked or answered. It was purely made to increase the amount of data online about the kinematics and kinetics of running. Thus, research conducted tested a multitude of variables, but for this paper only a handful are needed.

Researchers wishing to conduct a similar type of study are high encouraged to visit the detailed methodology here: https://peerj.com/articles/3298

\section{Definitions}

Flexibility variables (measured with a magnetic angle locator in degrees; see below for specifications)

RThomas: angle of the right thigh relative to a horizontal plane using the Thomas' test.

LThomas: angle of the left thigh relative to a horizontal plane using the Thomas' test.

ROber: angle of the right thigh relative to a horizontal plane using the Ober's test.

LOber: angle of the left thigh relative to a horizontal plane using the Ober's test.

Strength variables (measured with a hand-held dynamometer in kilograms (HHD); see below for specifications)

RHIPABD: MIVC (average maximal isometric voluntary contraction) of the right hip abductors.

LHIPABD: MIVC of the left hip abductors.

RHIPEXT: MIVC of the right hip extensors.

LHIPEXT: MIVC of the left hip extensors. 
RHIPER: MIVC of the right hip external rotators.

LHIPER: MIVC of the left hip external rotators.

Magnetic angle locator specifications: Model 700; Johnson Level \& Tool Mfg. Co., Inc., Mequon, WI, USA.

Dynamometer specifications: range: $0-1,330 \mathrm{~N}$; accuracy: $\pm 1 \%$; resolution: $1 \mathrm{~N}$; Nicholas MMT, Lafayette Instruments, Lafayette, IN, USA.

\section{Methods and Procedure continued}

Once the raw data files were downloaded, they were formatted to a simpler excel file that only contained the values of these 10 variables and the injury value (at this point, the injury value in the raw data either stated "yes" or "no". A simple algorithm to change "yes" and "no" to binary notation was used for ease later in the process).

Using the R programming language and R Studio, regression models were created to analyze and visualize the existing flexibility and relative muscle strength values against the existing injury values. Then, predictive analysis was conducted using a binary logistic regression structure.

Raw Data

\begin{tabular}{|c|c|c|c|c|c|c|c|c|c|c|c|}
\hline Injury & RThomas & LThomas & ROber & LOber & RHIPABD & LHIPABD & RHIPEXT & LHIPEXT & RHIPER & LHIPER & RHIPIR \\
\hline 1 & 10 & 11 & 43 & 40 & 16.8 & 21.575 & 16.575 & 21.675 & 9.66666667 & 7.25 & 10.9666667 \\
\hline 1 & 15 & 12 & 43 & 38 & 23 & 28.025 & 23.7 & 21.5 & 10.65 & 9.6 & 18.46666667 \\
\hline 1 & 19 & 15 & 44 & 41 & 22.0333333 & 24.3 & 23.325 & 19.05 & 10.3333333 & 10.7333333 & 15.5 \\
\hline 0 & 8 & 11 & 34 & 32 & 18.3 & 14.35 & 15.6 & 16.9666667 & 9.1 & 6.966666667 & 12.0666667 \\
\hline 1 & -1 & 4 & 33 & 29 & 18.8333333 & 25.3 & 20.325 & 19.9 & 12.2 & 7.375 & 10.7 \\
\hline 1 & 14 & 15 & 34 & 31 & 22.8333333 & 19.3 & 24.35 & 20.2 & 10.7 & 10.2333333 & 14.4 \\
\hline 0 & 25 & 10 & 32 & 29 & 26.7 & 31.2666667 & 27.2666667 & 26.3333333 & 12.3333333 & 12.7333333 & 19.5 \\
\hline 1 & 22 & 16 & 31 & 27 & 30.8 & 25.16666667 & 18.3 & 17.325 & 9.56666667 & 11.4 & 13.775 \\
\hline 0 & 35 & 40 & 25 & 45 & 33.66666667 & 34.625 & 23.825 & 22.1 & 10.7666667 & 13.8 & 18.4333333 \\
\hline 1 & 16 & 16 & 29 & 28 & 32.3333333 & 31.76666667 & 20.3666667 & 24.1333333 & 10.5 & 10.7666667 & 11.8 \\
\hline 0 & 9 & 9 & 32 & 32 & 23.1 & 21.3333333 & 21.8333333 & 20.56666667 & 13.9333333 & 12.4333333 & 17.325 \\
\hline 0 & 14 & 12 & 37 & 34 & 22.26666667 & 19.4333333 & 22.6333333 & 22.26666667 & 10.6666667 & 11.6 & 12.1333333 \\
\hline 0 & 2 & -2 & 39 & 37 & 22.875 & 21.7 & 15.1666667 & 16.575 & 9.45 & 9.475 & 11.3666667 \\
\hline 1 & 4 & 3 & 27 & 27 & 18.975 & 17.05 & 23.3333333 & 21.4 & 9.53333333 & 9 & 10.6 \\
\hline 0 & 10 & 9 & 32 & 34 & 26.4 & 26.2 & 19.3666667 & 19.3333333 & 7.925 & 7.53333333 & 13.8 \\
\hline 0 & 6 & 5 & 30 & 28 & 32.66666667 & 35.2 & 18.925 & 20.9 & 12.7 & 12.76666667 & 18.0666667 \\
\hline 1 & 20 & 22 & 32 & 32 & 29.6333333 & 26.775 & 21.1666667 & 21.5 & 11.0333333 & 9.575 & 15.025 \\
\hline 0 & 15 & 17 & 37 & 35 & 22.525 & 21.4333333 & 14.3333333 & 12.2 & 7.73333333 & 7.36666667 & 7.725 \\
\hline 0 & 5 & 5 & 31 & 30 & 28.9 & 30.4 & 24.06666667 & 24.3 & 12.5333333 & 11.0666667 & 16.825 \\
\hline 0 & 9 & 8 & 26 & 30 & 28.8333333 & 28.55 & 20.7 & 19.3666667 & 11.8333333 & 8.966666667 & 11.4 \\
\hline 0 & 5 & 5 & 34 & 32 & 39.1 & 36.96666667 & 29.475 & 28.66666667 & 12.7 & 10.5333333 & 13.1 \\
\hline 0 & 15 & 12 & 31 & 29 & 32.9333333 & 34.5333333 & 17.7666667 & 17.8 & 9.175 & 9.4 & 10.1 \\
\hline 0 & -1 & 1 & 34 & 34 & 45.76666667 & 34.1666667 & 33.95 & 29 & 13.1 & 14.575 & 19.9 \\
\hline 0 & 4 & 3 & 36 & 33 & 25.175 & 27.625 & 23.5 & 20.5333333 & 10.9 & 9.666666667 & 14.06666667 \\
\hline 0 & 6 & 6 & 28 & 25 & 28.5333333 & 29.76666667 & 20.875 & 21.65 & 13.1 & 10.8333333 & 13.2666667 \\
\hline 0 & 9 & 12 & 35 & 34 & 25.76666667 & 23.35 & 31.96666667 & 31.0333333 & 11.9 & 10.6333333 & 15.375 \\
\hline 0 & 11 & 11 & 30 & 28 & 18.75 & 21.6 & 26.625 & 26.075 & 12.525 & 11.625 & 10.125 \\
\hline
\end{tabular}




\section{Results}

Existing Variables Plots (Strength or Flexibility Value Against Injury Value)
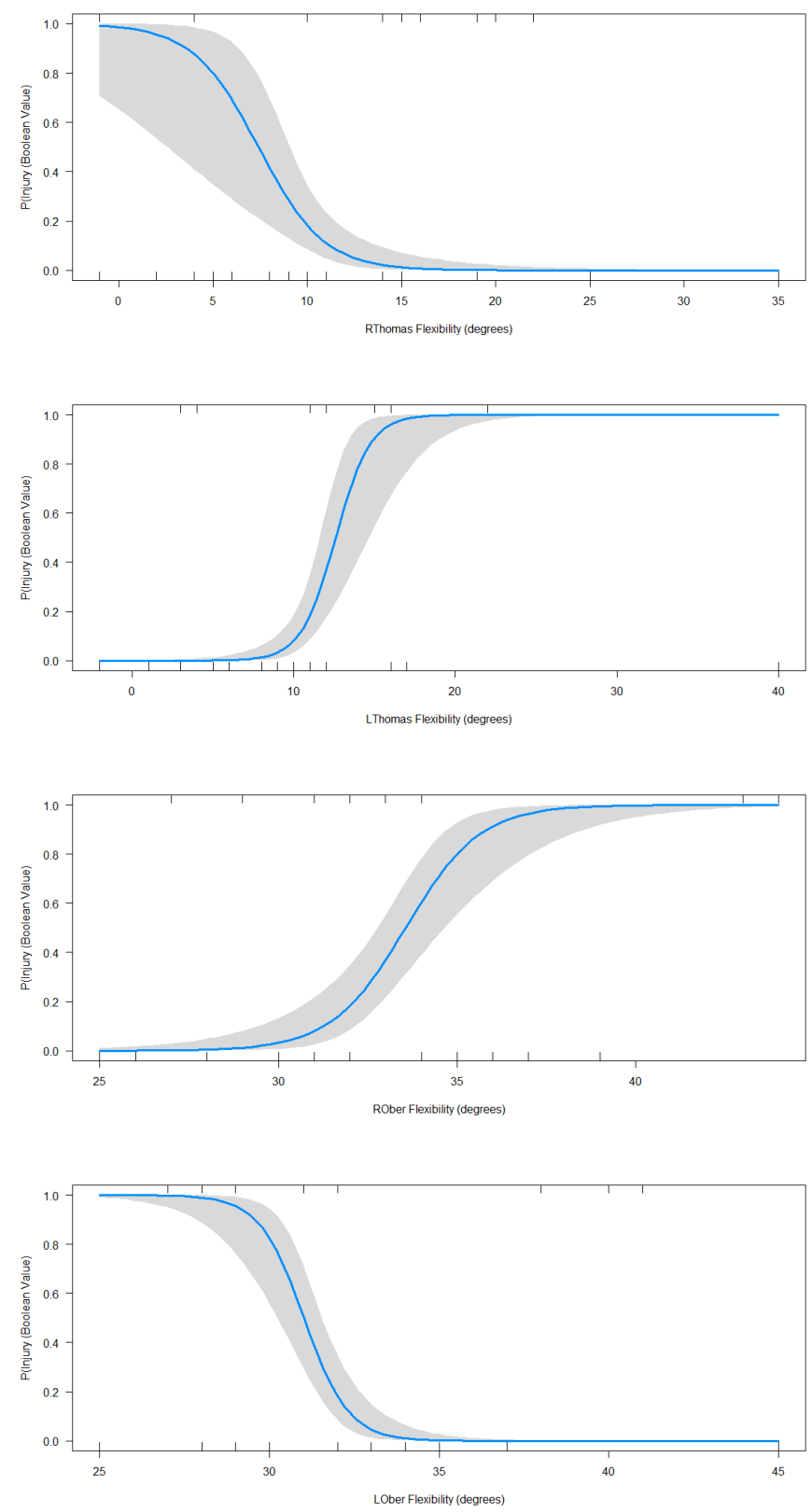

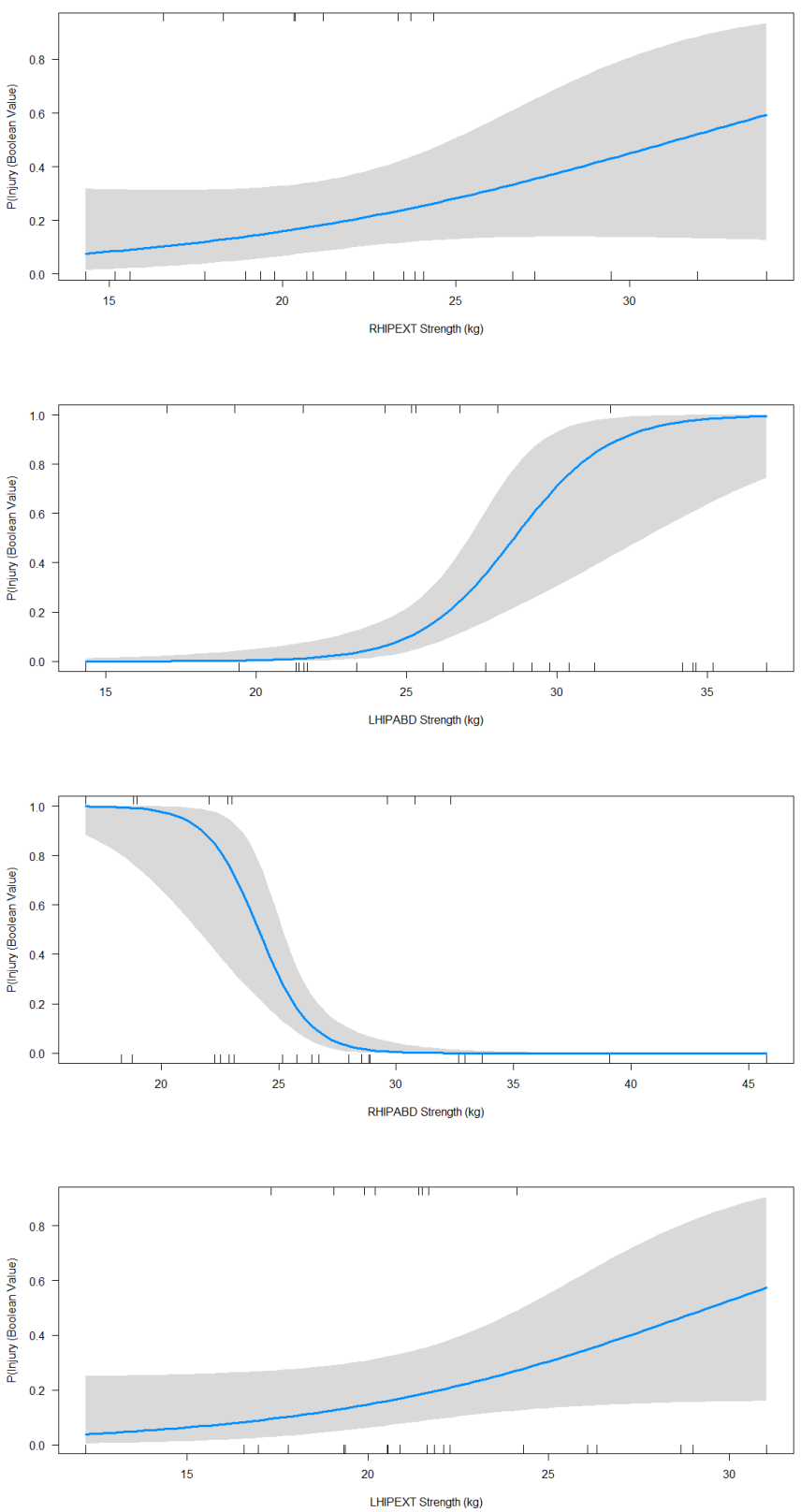

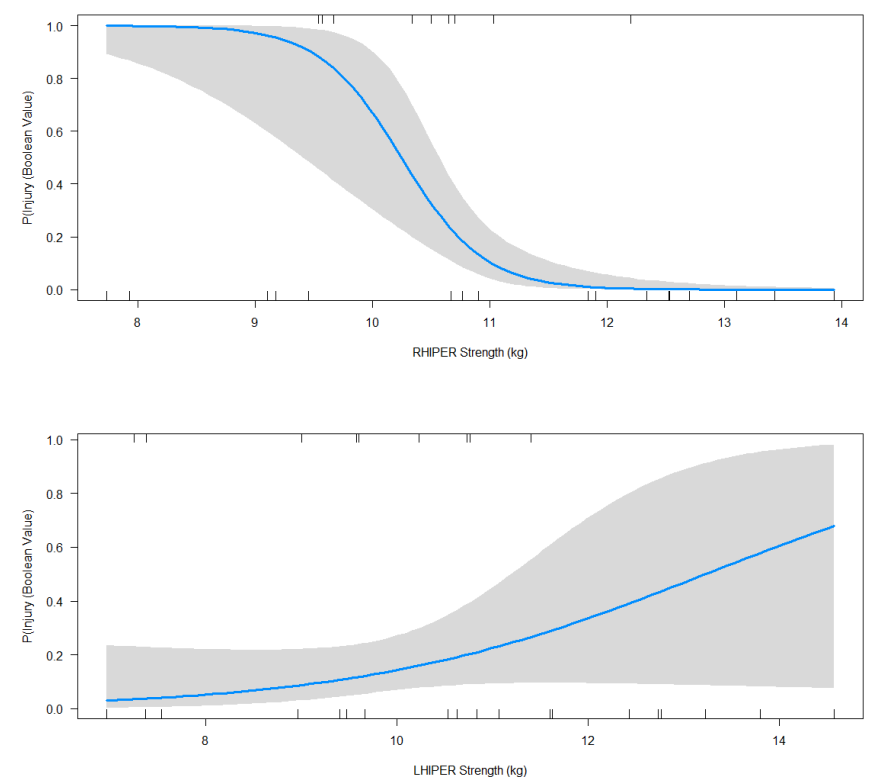

Predictive Results

\begin{tabular}{|l|r|r|r|r|r|}
\hline & B & \multicolumn{1}{|c|}{ S.E } & df & Sig. & \multicolumn{2}{|c|}{$\operatorname{Exp(B)}$} \\
\hline RThomas & -0.638 & 0.156 & 1 & 0 & 0.528 \\
\hline LThomas & 1.037 & 0.21 & 1 & 0 & 2.822 \\
\hline ROber & 0.97 & 0.204 & 1 & 0 & 2.638 \\
\hline LOber & -1.582 & 0.307 & 1 & 0 & 0.206 \\
\hline RHIPABD & -0.999 & 0.231 & 1 & 0 & 0.368 \\
\hline LHIPABD & 0.696 & 0.172 & 1 & 0 & 2.006 \\
\hline RHIPEXT & 0.105 & 0.86 & 1 & 0.222 & 1.111 \\
\hline LHIPEXT & 0.206 & 0.88 & 1 & 0.02 & 1.229 \\
\hline RHIPER & -3.01 & 0.74 & 1 & 0 & 0.49 \\
\hline LHIPER & 0.694 & 0.317 & 1 & 0.29 & 2.001 \\
\hline Constant & 26.377 & 6.113 & 1 & 0 & $2.85535 E+12$ \\
\hline
\end{tabular}

Definitions

B: slope of the model

S. E: Standard error df: degree of freedom

Sig.: $\mathrm{p}$ value

$\operatorname{Exp}(B)$ : exponent/factor

\section{Discussion}

Since injury is a concept that is tightly knitted to many uncontrollable variables, it impossible to create a closed environment that can accurately collect and analyze all possible outcomes of injury; however, one may see strength and flexibility as one of the leading controllable factors of injury proneness. Furthermore, strength and flexibility are 
values that the athlete themselves may control (with exceptions), thus allowing for the variability of injury controllable to some degree upon the athlete's wish.

In the visualization and analysis of the existing variables, it is interesting to see the variation in the correlation between the muscle groups and injury. Some muscle groups have a significant correlation with injury while other muscle groups have a low and even negative correlations with injury. In the case of having negative correlation, it is important to note that a visual negative relationship with flexibility and injury results in a positive correlation because the larger the angle the more flexible the subject. If one were to observe the slope of the predictive results in both the RThomas and LThomas variable, the slopes are extremely different. Due to the significant number of negative slopes, it is reasonable to infer the absolute value of the slopes depict the intensity of correlation from each muscle group against injury. Therefore, with this reasoning, one may conclude that the RThomas and LThomas variables contain a stronger correlation to injury in runners than variables such as the RHIPEXT and the LHIPEXT which both have positive but relatively small slopes. In this specific example, if one were to expand the variables and their meaning, we may see that the RThomas and LThomas variables depict the flexibility of a subject's hip flexors and their angle to a horizontal plane. The RHIPEXT and LHIPEXT variables both look at the average maximal isometric voluntary contraction of the right and left hip extensors. Despite the extensive use of the hip abductors, the hip flexors along with a couple other muscle groups are what drive the leg to create the stride. Thus, one can confirm relative consistency of both existing and predicted values.

\section{Conclusion}

Through the visualization of the existing variables as well as the predictive values, it is evident that there are a few errors in the consistency of the raw data and the predictive analysis. This is because, as stated prior, injury may happen through an almost infinite number of variables ranging from how well one ensures proper practice to staying healthy to the unluckiness of stepping on an abnormally angled object with enough force and impact to tear a muscle. This is extremely important to note, because the information given crafts a different lens for athletes today to injury. Nonetheless, the predictive analysis conducted still displays significant information regarding which muscle group affects injury in running. With this data, one may further their understanding of significant muscle groups that have a larger impact to injury in running, thus allowing the athlete to properly and efficiently control the variables that they may.

\section{Acknowledgments}

I would like to thank my advisor Alfred Renaud for helping me with this project.

\section{References}

Fukuchi, R. K. Fukuchi, C. A., \& Duarte, M. (2017). A public dataset of running biomechanics and the effects of running speed on lower extremity kinematics and kinetics. PeerJ 5, e3298. https://doi.org/10.7717/peerj.3298

Roger A. Mann, J. (1980). Biomechanics of walking, running, and sprinting - Roger A. Mann, John Hagy, 1980. Retrieved August 31, 2020, from https://journals.sagepub.com/doi/pdf/10.1177/036354658000800510

Dunne, J. (2019, June 10). Key Muscles Used in Running: 5 Main Muscle Groups. Retrieved August 31, 2020, from https://www.kinetic-revolution.com/key-muscle-groups-for-stronger-running/ 